\title{
ON WEAKLY PERIODIC-LIKE RINGS AND COMMUTATIVITY THEOREMS
}

\author{
HAZAR ABU-KHUZAM, HOWARD E. BELL AND ADIL YAQUB
}

\begin{abstract}
A ring $R$ is called periodic if, for every $x$ in $R$, there exist distinct positive integers $m$ and $n$ such that $x^{m}=x^{n}$. An element $x$ of $R$ is called potent if $x^{k}=x$ for some integer $k>1$. A ring $R$ is called weakly periodic if every $x$ in $R$ can be written in the form $x=a+b$ for some nilpotent element $a$ and some potent element $b$ in $R$. A ring $R$ is called weakly periodic-like if every element $x$ in $R$ which is not in the center $C$ of $R$ can be written in the form $x=a+b$, with $a$ nilpotent and $b$ potent. Some structure and commutativity theorems are established for weakly periodic-like rings $R$ satisfying certain torsion-freeness hypotheses along with conditions involving some elements being central.
\end{abstract}

Throughout, $R$ is an associative ring, $N$ is the set of nilpotent elements of $R, C$ is the center of $R, C(R)$ is the commutator ideal of $R,[x, y]$ denotes the usual commutator $x y-y x$, and $J$ denotes the Jacobson radical of $R$. We now state formally the definition of a weakly periodic-like ring.

Definition 1. A ring $R$ is called weakly periodic-like if every $x$ in $R \backslash C$ can be written in the form

$$
x=a+b, \quad a \in N, \quad b \text { potent }\left(b^{k}=b \text { for some } k>1\right), \quad x \in R \backslash C .
$$

In preparation for the proofs of the main theorems, we state the following known lemmas.

Lemma 1.([3]) If $[x, y]$ commutes with $x$, then $\left[x^{k}, y\right]=k x^{k-1}[x, y]$ for all positive integers $k$.

Lemma 2.([10]) Suppose $R$ is a ring with identity 1 . If $x^{m}[x, y]=0$ and $(x+$ $1)^{m}[x, y]=0$ for some $x, y$ in $R$ and some positive integer $m$, then $[x, y]=0$. A similar statement holds if we assume $[x, y] x^{m}=0$ and $[x, y](x+1)^{m}=0$ instead.

Lemma 3.([4]) Let $R$ be an $n$-torsion-free ring with identity such that $\left[x^{n}, y^{n}\right]=0$ for all $x, y$ in $R$. Let $N$ denote the set of nilpotent elements of $R$. Then

Received April 8, 2005.

2000 Mathematics Subject Classification. Primary 16 U80.

Key words and phrases. Periodic ring, weakly periodic ring, weakly periodic-like ring, potent element, commutator ideal, Jacobson radical, semisimple ring, primitive ring, subdirect sum. 
(i) $a \in N, x \in R$ imply $\left[a, x^{n}\right]=0$.

(ii) $a \in N, b \in N$ imply $[a, b]=0$.

(iii) If $N$ is contained in the center of $R$, then $R$ is commutative.

Lemma 4.([1]) Suppose $R$ is a ring with center $C$ and $N$ is the set of nilpotent elements of $R$. Suppose that (a) $N$ is commutative; (b) for all a in $N$ and all $x$ in $R$, ax $-x a$ commutes with $x$; $(c)$ for all $x \in R$, we have $x \in C$ or $x^{n}-x \in N$ for some $n=n(x)>1$. Then $R$ is commutative.

Lemma 5.([6]) Suppose $R$ is a ring such that for all $x, y$ in $R,\left[x^{n}, y^{n}\right]=0$, where $n$ is a fixed positive integer. Then the commutator ideal of $R$ is nil, and hence $N$ is an ideal of $R$.

Lemma 6.([7]) Suppose $R$ is a ring such that for every $x$ in $R$, there exists an integer $n=n(x)>1$ such that $x-x^{n}$ is the center of $R$. Then $R$ is commutative.

Lemma 7.([9]) Suppose $R$ is a ring with identity and $m$ and $n$ are fixed positive integers which are relatively prime. Suppose that, for all $x, y$ in $R$,

$$
\left[x^{n}, y^{n}\right]=0 \quad \text { and } \quad\left[x^{m}, y^{m}\right]=0 .
$$

Then $R$ is commutative.

Lemma 8.([11]) Let $R$ be a weakly periodic-like ring, $J$ the Jacobson radical of $R$, and $C(R)$ the commutator ideal of $R$. IF $C(R) \subseteq J$, then the set $N$ of nilpotents is an ideal and $R / N$ is commutative.

We are now in a position to prove our main theorems. In the first theorem, we do not assume that the ground ring $R$ is weakly periodic-like.

Theorem 1. Let $R$ be a ring with identity 1 and let $n>1$ be a fixed integer. Suppose $R$ is $n$-torsion-free and satisfies $\left[x^{n}, y^{n}\right]=0$ for all $x, y$ in $R$. Suppose, further, that for all $x, y$ in $R$, either $(x y)^{n+1}-(y x)^{n+1} \in C$ or $(x y)^{n-1}-y^{n-1} x^{n-1} \in C$, where $C$ is the center of $R$. Then $R$ is commutative.

Proof. By Lemma 3, the set $N$ of nilpotent elements of $R$ is commutative and

$$
\left[a, b^{n}\right]=0 \text { for all } a \text { in } N \text { and all } b \text { in } R \text {. }
$$

Moreover, by Lemma 5, $N$ is an ideal of $R$ and hence (since $N$ is commutative)

$$
N^{2} \subseteq C \quad(C=\text { center of } R) .
$$

Let $a \in N, b \in R$. We now consider the two possibilities in the hypothesis of the theorem.

CASE 1. $(x y)^{n+1}-(y x)^{n+1} \in C$. 
In this case,

$$
((a+1) b)^{n+1}-(b(a+1))^{n+1} \in C .
$$

Since $N$ is a commutative ideal of $R$ and $N^{2} \subseteq C$, a close look at (4) shows that

$$
\left[a, b^{n+1}\right] \in C
$$

and hence

$$
\left[a, b^{n+1}\right]=[a, b] b^{n}+b\left[a, b^{n}\right] \in C .
$$

But, by (2), $\left[a, b^{n}\right]=0$ and hence (6) implies that $[a, b] b^{n} \in C$, which, in turn, implies that $\left[[a, b] b^{n}, b\right]=0$ and hence

$$
[[a, b] b] b^{n}=0, \quad(a \in N, b \in R) .
$$

CASE 2. $(x y)^{n-1}-y^{n-1} x^{n-1} \in C$.

As shown above, we have

$N$ is a commutative ideal, $N^{2} \subseteq C,\left[a, b^{n}\right]=0$ for all $a$ in $N, b$ in $R$.

We may assume $((a+1) b)^{n+1}-(b(a+1))^{n+1}$ is not in $C$, and hence $(b(a+1))^{n+1}-$ $((a+1) b)^{n+1}$ is not in $C$. Therefore,

$$
((a+1) b)^{n-1}-b^{n-1}(a+1)^{n-1} \in C
$$

and

$$
(b(a+1))^{n-1}-(a+1)^{n-1} b^{n-1} \in C .
$$

By subtracting the two expressions in (9) and (10), and using the facts in (8), it can be seen that

$$
\left[a, b^{n-1}\right]-\left\{-(n-1)\left[a, b^{n-1}\right]\right\} \in C,
$$

and hence (since $R$ is $n$-torsion-free)

$$
\left[a, b^{n-1}\right] \in C \text { for all } a \text { in } N \text { and all } b \text { in } R .
$$

Moreover, since $\left[a, b^{n}\right]=0$ (see $\left.(8)\right)$, we have

$$
0=\left[a, b^{n}\right]=[a, b] b^{n-1}+b\left[a, b^{n-1}\right],
$$

and hence

$$
[a, b] b^{n-1}=-b\left[a, b^{n-1}\right] .
$$

By (11), the right side of (13) commutes with $b$, and hence, $[a, b] b^{n-1}$ commutes with $b$. Thus,

$$
\left[[a, b] b^{n-1}, b\right]=0
$$


and hence

$$
[a, b] b^{n}-b[a, b] b^{n-1}=0,
$$

which implies

$$
\{[a, b] b-b[a, b]\} b^{n-1}=0 .
$$

Therefore, $[[a, b], b] b^{n-1}=0$, and hence

$$
[[a, b], b] b^{n}=0, \quad(a \in N, b \in R) .
$$

In view of (7) and (14), we see that

$$
[[a, b] b] b^{n}=0 \text { in both cases, } \quad(a \in N, b \in R) .
$$

Replacing $b$ by $b+1$ in the aove argument shows that (see (15))

$$
[[a, b], b](b+1)^{n}=0 .
$$

Combining (15), (16), and Lemma 2, we get

$$
[[a, b], b]=0, \quad(a \in N, b \in R) .
$$

By $(2),\left[a, b^{n}\right]=0$, which when combined with (17) and Lemma 1 yields $n b^{n-1}[a, b]=0$. Since $R$ is $n$-torsion-free, it follows that

$$
b^{n-1}[a, b]=0, \quad(a \in N, b \in R) .
$$

Replacing $b$ by $b+1$ in the above argument, we see that (see (18))

$$
(b+1)^{n-1}[a, b]=0, \quad(a \in N, b \in R) .
$$

Combining (18), (19), and Lemma 2, we otain

$$
[a, b]=0 \text { for all } a \in N \text { and all } b \in R \text {. }
$$

The theorem now follows from (20) and Lemma 3(iii).

We now turn our attention to weakly periodic-like rings. We begin with the following main structure theorem.

Theorem 2. Let $R$ be a weakly periodic-like ring (not necessarily with identity), and let $N$ be the set of nilpotent elements of $R$. Suppose $P$ is a ring property which is satisfied by every subring $S$ of $R$ and also by every homomorphic image of the subring $S$. Suppose, further, that $P$ is not satisfied by any complete matrix ring of $n \times n$ matrices over any division ring, where $n>1$. Then

(i) $N$ is an ideal of $R$. 
(ii) $R / N$ is commutative.

(iii) If $x$ is in $R$, then either $x \in C$ or $x-x^{m} \in N$ for some integer $m>1$.

Proof. Let $J$ be the Jacobson radical of $R$. Then

$$
R / J \cong \text { a subdirect sum of primitive rings } R_{i}, \quad i \in \Gamma \text {. }
$$

In view of Jacobson's density theorem [8; p.33], the ring property $P$ hypothesis guarantees that each primitive ring $R_{i}$ in (21) nust be a division ring, and hence (21) yields

$$
R / J \cong \text { a subdirect sum of division rings } R_{i}, \quad i \in \Gamma \text {. }
$$

Furthermore, since the homomorphic image of a weakly periodic-like ring is also weakly periodic-like, each division ring $R_{i}$ in $(22)$ is weakly periodic-like. Hence (see Definition 1 ), for any $x_{i}$ in $R_{i}$, we have

$$
x_{i} \in \text { Center of } R_{i} \text { or } x_{i} \text { is potent }\left(x_{i}^{k}=x_{i} \text { for some } k>1\right) .
$$

Hence, for any $x_{i}$ in $R_{i}, x_{i}-x_{i}^{k} \in$ Center of $R_{i}$, where $k>1$, and thus by Lemma $6, R_{i}$ is commutative. Hence, by $(22), R / J$ is commutative, and thus

$$
C(R) \subseteq J \quad(C(R) \text { denotes the commutator ideal of } R) .
$$

Parts (i) and (ii) of the theorem now follow from (24) and Lemma 8.

To prove part (iii), suppose $x \in R \backslash C$. Then, by Definition 1 ,

$$
x=a+b ; a \in N, b \text { potent satisfying } b^{m}=b, m>1 .
$$

Hence,

$$
x-a=b=b^{m}=(x-a)^{m},
$$

which implies that $x-x^{m} \in N$, since $N$ is an ideal (see part(i)). This proves part (iii), and the theorem is proved.

The following lemma will be needed for the proofs of the remaining theorems.

Lemma 9. Let $R$ be a weakly periodic-like ring with the set of nilpotents commutative and with idempotents central. If $R$ has a property which implies commutativity in weakly periodic-like rings with identity and which is inherited by ideals, then $R$ is commutative.

Proof. First, we prove that the set $P_{0}$ of potent elements is central. Suppose

$$
a \in P_{0} \text { with } a^{n}=a, \quad n>1 .
$$

Let $e=a^{n-1}$. Then, since $e$ is central idempotent, $e R$ is a ring with identity. Indeed, $e R$ is weakly periodic-like which, in fact, is an ideal of $R$. The hypothesis of the lemma (referring to the property inheritance) guarantees that

$$
e R \text { is commutative. }
$$


Therefore,

$$
(e a)(e x)=(e x)(e a) \text { for all } x \text { in } R .
$$

Recall that $e=a^{n-1}$ is a central idempotent element of $R$, and hence (27) implies that

$$
e a x=e x a=x a e ; \text { that is, } a^{n-1} a x=x a a^{n-1} \text {, or } a^{n} x=x a^{n} \text {, }
$$

and thus, by (25), $a x=x a$ for all $x$ in $R$. This proves that

The set $P_{0}$ of potent elements of $R$ is central.

To complete the proof, suppose $x, y \in R$. If $x \in C$ or $y \in C$, then clearly $[x, y]=0$. So suppose $x \notin C$ and $y \notin C$. Then, by Definition 1 ,

$$
x=a+b, y=a^{\prime}+b^{\prime} ; a, a^{\prime} \text { nilpotent and } b, b^{\prime} \text { potent. }
$$

By (28), $b$ and $b^{\prime}$ are central, and hence

$$
[x, y]=\left[a+b, a^{\prime}+b^{\prime}\right]=\left[a, a^{\prime}\right]=0,(\text { since } N \text { is commutative) } .
$$

This proves the lemma.

Next, we prove

Theorem 3. Let $R$ be a weakly periodic-like ring, and let $n$ be a fixed positive integer. Suppose $R$ is $n$-torsion-free and, for all $x, y$ in $R,(x y)^{n}-(y x)^{n} \in C$ (the center of $R$ ). Suppose, further, that the set $N$ of nilpotents is commutative. Then, $R$ is commutative.

Proof. Let $P$ be the ring property " $(x y)^{n}-(y x)^{n}$ is always central." Clearly, this property is satisfied by all subrings and all homomorphic images of any subring of $R$. Moreover, this property $P$ is not satisfied by any complete matrix ring $D_{n}$ of $n \times n$ matrices over any division ring $D$, where $n>1$, as can be seen by taking $x$ and $y$ (in $\left.D_{n}\right)$ to be

$$
x=E_{11}, \quad y=E_{11}+E_{12}, \quad\left(E_{11}, E_{12} \in D_{n}\right) .
$$

Hence, by Theorem 2(iii), we have

$$
\text { If } x \in R \backslash C \text {, then } x-x^{m} \in N \text { for some integer } m>1 \text {. }
$$

Moreover, by Theorem 2(i), $N$ is an ideal which is commutative (by hypothesis). The net result is:

$$
N \text { is a commutative ideal and hence } N^{2} \subseteq C \text {. }
$$

We now distinguish two cases

CASE 1. $1 \in R$. Suppose $a \in N, b \in R$. Then, by hypothesis,

$$
((a+1) b)^{n}-(b(a+1))^{n} \in C .
$$


In view of (31), an easy argument shows that (32) implies

$$
\left[a, b^{n}\right] \in C \quad(a \in N, b \in R) .
$$

Let $S$ be the subring of $R$ generated by the set of all elements $x^{n}, x \in R$. By Theorem 2 (ii), $R / N$ is commutative, and hence

$$
\left(x_{1} \cdots x_{k}\right)^{n}-x_{1}^{n} \cdots x_{k}^{n} \in N \text { for all } x_{1}, \ldots, x_{k} \text { in } R .
$$

Since $N$ is commutative (see (31)), therefore

$$
\left[a,\left(x_{1} \ldots x_{k}\right)^{n}\right]=\left[a, x_{1}^{n} \ldots x_{k}^{n}\right], \quad(a \in N) .
$$

Moreover, by (33), the commutator on the left side of (34) is central, and hence

$$
\left[a, x_{1}^{n} \ldots x_{k}^{n}\right] \in C \text { for all } x_{1}, \ldots, x_{k} \text { in } R .
$$

Since the commutator is linear with respect to the second argument (in particular), (35) readily implies that

$$
[a, x] \in C(S) \text { for all } a \in N(S) \text { and all } x \in S,
$$

where $N(S)$ and $C(S)$ denote the set of nilpotents of $S$ and the center of $S$, respectively. Also, by Theorem 2(iii), we have:

$$
\text { If } x \in S \text {, then } x \in C(S) \text { or } x-x^{m} \in N(S) \text { for some } m>1 \text {. }
$$

Moreover, by (31),

$$
N(S) \text { is commutative. }
$$

Combining (36), (37), (38), and Lemma 4, we conclude that $S$ is commutative, and hence

$$
\left[x^{n}, y^{n}\right]=0 \text { for all } x, y \text { in } R .
$$

Since $R$ is $n$-torsion-free and satisfies (39), therefore by Lemma 3(i),

$$
\left[a, x^{n}\right]=0 \text { for all } a \in N, x \in R \text {. }
$$

Let $a \in N, u=1+a$. Then, by (40)

$$
\left[u, x^{n}\right]=0, \quad(u=1+a, a \in N) .
$$

By hypothesis,

$$
\left(\left(x^{n-1} u\right) x\right)^{n}-\left(x\left(x^{n-1} u\right)\right)^{n} \in C
$$

and hence

$$
x^{n-1} u x^{n} u x^{n} \cdots u x^{n}(u x)-\left(x^{n} u\right)^{n} \in C .
$$


Combining (41) and (42), we obtain

$$
x^{n^{2}-1} u^{n} x-x^{n^{2}} u^{n} \in C,
$$

and hence

$$
x^{n^{2}-1}\left[u^{n}, x\right] \in C, \quad(u=1+a, a \in N) .
$$

By $(31), N^{2} \subseteq C$, and hence

$$
\left[u^{n}, x\right]=\left[(1+a)^{n}, x\right]=[n a, x]=n[a, x] .
$$

Combining (43), (44), we obtain

$$
n x^{n^{2}-1}[a, x] \in C, \quad(a \in N, x \in R) .
$$

Since $R$ is $n$-torsion-free, this implies that $x^{n^{2}-1}[a, x] \in C$, and hence

$$
\left[x^{n^{2}-1}[a, x], x\right]=0,
$$

which implies

$$
x^{n^{2}-1}[[a, x], x]=0, \quad(a \in N, x \in R) .
$$

Replacing $x$ by $x+1$ in the above argument, we get

$$
(x+1)^{n^{2}-1}[[a, x], x]=0, \quad(a \in N, x \in R) .
$$

Combining (45), (46), and Lemma 2, we conclude that

$$
[[a, x], x]=0, \quad(a \in N, x \in R) .
$$

The theorem now follows from (30), (47), and the hypothesis that $N$ is commutative (see Lemma 4).

CASE 2. $R$ does not have an identity. In this case, we first prove the following:

CLAIM. The idempotents of $R$ are central. Let $e^{2}=e \in R$. By hypothesis,

$$
(e(e+e r-e r e))^{n}-((e+e r-e r e) e)^{n} \in C,
$$

and hence $e r-e r e \in C$. Therefore,

$$
e r-e r e=e(e r-e r e)=(e r-e r e) e=0,
$$

and thus er $=e r e$. Similarly, $r e=e r e$, and the claim follows.

Since the properties of torsion-freeness and " $(x y)^{n}-(y x)^{n}$ is always central" are inherited by any ideal of $R$, the theorem now follows from Case 1 and Lemma 9. This completes the proof. 

3.

The following result was proved by the authors in [2], and is a corollary of Theorem

Corollary 1. Let $n$ be a fixed positive integer and let $R$ be an $n$-torsion-free periodic ring such that $(x y)^{n}-(y x)^{n}$ is central for all $x, y$ in $R$. If the set $N$ of nilpotents of $R$ is commutative, then $R$ is commutative.

This follows from Theorem 3, since a periodic ring is weakly periodic [5], and hence is also weakly periodic-like.

Theorem 4. Suppose $R$ is a weakly periodic-like ring, and suppose $m$ and $n$ are positive integers which are relatively prime. Suppose that

$$
(x y)^{n}-(y x)^{n} \in C \text { and }(x y)^{m}-(y x)^{m} \in C \quad(C \text { is center of } R) .
$$

Suppose, further, that the set $N$ of nilpotents is commutative, Then $R$ is commutative.

Proof. A careful examination of the proof of Theorem 3 shows that the hypothesis that $R$ is $n$-torsion-free was not used in the proof of (31), and thus

$$
N \text { is a commutative ideal of } R \text { and } N^{2} \subseteq C \text {. }
$$

CASE 1. $1 \in R$. Again, the proof of (39) did not use the hypopthesis that $R$ is $n$-torsion-free, and hence

$$
\left[x^{n}, y^{n}\right]=0 \text { for all } x, y \text { in } R .
$$

Similarly, by making use of the hypothesis " $(x y)^{m}-(y x)^{m}$ is central", we see that (see (49))

$$
\left[x^{m}, y^{m}\right]=0 \text { for all } x, y \text { in } R .
$$

Hence, by (49), (50), and Lemma 7, we conclude that $R$ is commutative (recall that $1 \in R)$.

CASE 2. $1 \notin R$. Using the argument in the proof of the Claim of Theorem 3 , we see that

The idempotents of $R$ are central.

As indicated in the proof of Case 2 of Theorem 3, the theorem now follows from Case 1 and Lemma 9. This completes the proof.

Recalling that a periodic ring is weakly periodic-like [5], and taking $m=1, n=1$ in Theorem 4, we obtain the following

Corollary 2. A periodic ring with commuting nilpotents and central commutators is commutative.

Theorem 5. Suppose $R$ is a weakly periodic-like ring, and suppose $m$ and $n$ are positive integers which are relatively prime. Suppose that $R$ is mn-torsion-free, and

$$
\left[x^{n}, y^{n}\right] \in C \text { and }\left[x^{m}, y^{m}\right] \in C \text { for all } x, y \text { in } R .
$$


Suppose, further, that the set $N$ of nilpotents is commutative. Then $R$ is commutative.

Proof. Let $P$ be the ring property " $\left[x^{n}, y^{n}\right] \in C$ for all $x, y$ in $R$." Clearly, this property is satisfied by all subrings and all homomorphic images of any subring of $R$. Moreover, this property $P$ is not satisfied by any complete matrix ring $D_{n}$ of $n \times n$ matrices over any division ring $D$, where $n>1$, as can be seen by taking

$$
x=E_{11}, \quad y=E_{11}+E_{12}, \quad\left(E_{11}, E_{12} \in D_{n}\right) .
$$

Hence, by Theorem 2(i),

$N$ is an ideal of $R, \quad$ ( $N$ is the set of nilpotents).

Moreover,

$$
N \text { is commutative, and hence } N^{2} \subseteq C \text { (see (52)). }
$$

We now distinguish two cases.

CASE 1. $1 \in R$. Let $a \in N, b \in R$. Then, $\left[(1+a)^{n}, b^{n}\right] \in C$, which when combined with (52) and (53) yields $n\left[a, b^{n}\right] \in C$. Since $R$ is $m n$-torsion-free,

$$
\left[a, b^{n}\right] \in C \text { for all } a \in N, b \in R .
$$

This is precisely (33) above. By repeating the argument used in (33) through (39) above, we conclude that

$$
\left[x^{n}, y^{n}\right]=0 \text { for all } x, y \text { in } R .
$$

Similarly, using the hypothesis $\left[x^{m}, y^{m}\right] \in C$, the above argument yields

$$
\left[x^{m}, y^{m}\right]=0 \text { for all } x, y \text { in } R .
$$

Hence, by (55), (56), and Lemma 7, we conclude that $R$ is commutative (recall that $1 \in R)$.

CASE 2. $1 \notin R$. First, note that the idempotents are central. To see this, let $e^{2}=e \in R, r \in R$. By hypothesis,

$$
\left[e^{n},(e+e r-e r e)^{n}\right] \in C,
$$

and hence $e r-e r e \in C$. Thus,

$$
e r-e r e=e(e r-e r e)=(e r-e r e) e=0,
$$

and hence $e r=e r e$. Similarly, $r e=e r e$, and hence

All idempotents of $R$ are central.

Arguing as we did in the proof of Case 2 of Theorem 3, the theorem now follows from Case 1 and Lemma 9. This completes the proof. 


\section{Acknowledgements}

Prof. Bell's research is supported by the Natural Sciences and Engineering Research Council of Canada, Grant No. 3961.

\section{References}

[1] H. Abu-Khuzam, A commutativity theorem for periodic rings, Math. Japon. 32 (1987), $1-3$.

[2] H. Abu-Khuzam, H. E. Bell and A. Yaqub, Commutativity of rings satisfying certain polynomial identities, Bull. Austral. Math. Soc. 44 (1991), 63-69.

[3] H. Abu-Khuzam and A. Yaqub, n-torsion-free rings with commuting powers, Math. Japon. 25 (1980), 37-42.

[4] H. E. Bell, On rings with commuting powers, Math. Japon. 24 (1979), 473-478.

[5] H. E. Bell, A commutativity study for periodic rings, Pacific J. Math. 70 (1977), 29-36.

[6] I. N. Herstein, A commutativity theorem, J. Algebra 38 (1976), 112-118.

[7] I. N. Herstein, A generalization of a theorem of Jacobson III, Amer. J. Math. 75 (1953), 105-111.

[8] N. Jacobson, Structure of Rings, Amer. Math. Soc. Colloq. Publications, Vol. 37, American Mathematical Society, Providence, RI, 1964.

[9] W. K. Nicholson and A. Yaqub, A commutativity theorem for rings and groups, Canad. Math. Bull. 22 (1979), 419-423.

[10] W. K. Nicholson and A. Yaqub, A commutativity theorem, Algebra Universalis 10 (1980), 260-263.

[11] A. Yaqub, Weakly periodic-like rings and commutativity, Studia Sci. Math. Hungar. 43(2006), 275-284.

Department of Mathematics, American University of Beirut, Beirut, Lebanon.

Department of Mathematics, Brock University, St. Catharines, Ontario, Canada L2S 3A1.

Department of Mathematics, University of California, Santa Barbara, CA 93106, USA. 